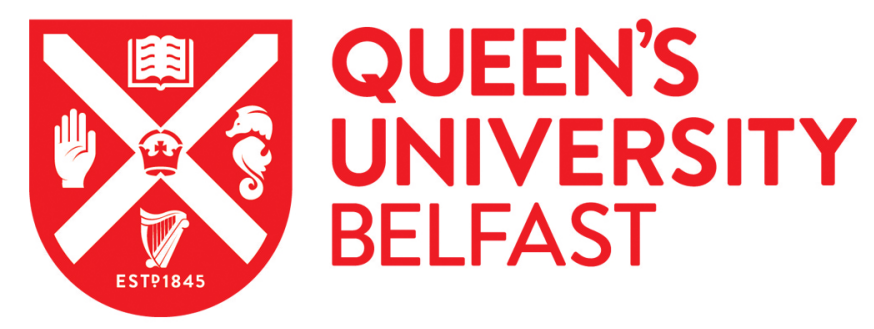

\title{
Relative resistance index (RRI) - a scoring system for antibiotic resistance in Pseudomonas aeruginosa
}

\author{
Ewing, J. C., McCaughan, J., Moore, J., Fairley, D., Sutherland, B., Reid, A., \& Downey, D. (2017). Relative \\ resistance index (RRI) - a scoring system for antibiotic resistance in Pseudomonas aeruginosa. British Journal of \\ Biomedical Science. https://doi.org/10.1080/09674845.2017.1338500
}

Published in:

British Journal of Biomedical Science

Document Version:

Peer reviewed version

Queen's University Belfast - Research Portal:

Link to publication record in Queen's University Belfast Research Portal

\begin{abstract}
General rights
Copyright for the publications made accessible via the Queen's University Belfast Research Portal is retained by the author(s) and / or other copyright owners and it is a condition of accessing these publications that users recognise and abide by the legal requirements associated with these rights.
\end{abstract}

Take down policy

The Research Portal is Queen's institutional repository that provides access to Queen's research output. Every effort has been made to ensure that content in the Research Portal does not infringe any person's rights, or applicable UK laws. If you discover content in the Research Portal that you believe breaches copyright or violates any law, please contact openaccess@qub.ac.uk. 


\section{BJBS 5616}

Relative Resistance Index (RRI) - a Scoring System for Antibiotic Resistance in

J Ewing 1, J McCaughan1, 2,3,4, J Moore ${ }^{1,3,4}$,

1. Regional Adult Cystic Fibrosis Centre, Belfast City Hospital, Belfast Health \& Social Care Trust, Northern Ireland Ireland

3. Department of Microbiology, Belfast Health \& Social Care Trust, Northern

2. Regional Paediatric Cystic Fibrosis Centre, Royal Belfast Hospital for Sick Children, Royal Victoria Hospital, Belfast Health \& Social Care Trust, Northern Ireland

\section{Corresponding author:}

Dr Damian G Downey, Regional Respiratory Centre

22 Ground Floor, Belfast City Hospital, Lisburn Road

23 Belfast, BT9 7AB, Northern Ireland, UK.

24 Email: d.downey@qub.ac.uk 
ABSTRACT

Background: There is a need to measure antibiotic resistance of Pseudomonas aeruginosa

(PA) in cystic fibrosis (CF), either qualitatively or quantitatively, to inform patient

management. The aim of this study was to develop a simple method by which resistance can be quantified by calculating a relative resistance index (RRI), and to assess correlation of RRIs with clinical variables.

Methods: In our model, RRIs were calculated based on resistance to aztreonam, ceftazidime, ciprofloxacin, colistin, meropenem, tazocin, temicillin and tobramycin. Eighty-five adults with CF and chronic PA colonisation were identified. For each, all PA cultures were allocated a score of 0 for susceptible, 0.5 for intermediate resistance or 1 for resistant for each antibiotic listed above, and the RRI calculated by dividing the sum of these by the number of antibiotics, giving a maximum score of 1 . The mean RRIs for all cultures were correlated with key clinical variables monitored in cystic fibrosis patients (including age, FEV $\mathrm{F}_{1}$, IV antibiotic days and BMI).

Results: RRIs for non-mucoid PA exhibited moderate positive correlation with total number of IV days $(\mathrm{r}=0.405 ; \mathrm{p}<0.001)$ and moderate negative correlation with $\mathrm{FEV}_{1} \%$ predicted $(\mathrm{r}=-0.437 ; \mathrm{p}<0.001)$. RRIs were not significantly correlated with duration of colonisation, typing (clonal vs other strain) or BMI. Median RRIs were significantly higher for females $(0.26$, IQR $0.13-0.54)$ than males $(0.18$, IQR $0.07-0.37)$ for non-mucoid PA only $(\mathrm{p}=$ $46 \quad 0.03)$.

Conclusions: RRI is an easily calculated measure that correlates with other clinical variables in cystic fibrosis patients and enables quantitative monitoring of resistance. 


\section{INTRODUCTION}

51 Cystic fibrosis (CF) is an autosomal recessive genetic disorder characterised by defective mucociliary clearance and chronic airway infection [1]. The most commonly isolated pathogen from $\mathrm{CF}$ airways is the opportunistic Gram-negative bacterium Pseudomonas aeruginosa (PA) [2]. Chronic PA infection is associated with significant morbidity and mortality in CF patients [3] and necessitates multiple antibiotic courses, which increase the risk of the development of antibiotic resistance [2]. Many of the PA strains isolated from CF airways are of the mucoid phenotype, capable of producing large quantities of alginate which enable the formation of a matrix in which microcolonies can exist. These microcolonies are thought to provide protection against the host immune response and antibiotic therapy; conversion of PA through mutation in patients to the mucoid phenotype is associated with increased morbidity and mortality [4]. Typically, initial colonisation is with non-mucoid forms of PA, with the mucoid form predominate later.

Antibiotic resistance in PA may be driven by inappropriate use of antibiotics in either oral, nebulised or intravenous formulations, as well as by the use of biocides which might also drive increased antibiotic resistance [5]. Development of novel virulence model systems in PA may help predict clinical outcomes in patients with CF [6].

There is a need to measure antibiotic susceptibility (AS) of PA, either qualitatively or quantitatively, to inform patient management. AS in PA is generally reported by classifying resistance to individual antibiotics qualitatively (sensitive (S), intermediate (I) or resistant (R)) or quantitatively (minimum inhibitory concentration (MIC)). PA strains can thus be grouped by the presence or absence of multi-drug resistance, the definition of which varies 
74 considerably in biomedical literature [7]. Multiple-antibiotic resistant PA has previously

75 been associated with a lower $\mathrm{FEV}_{1} \%$ predicted [8].

76

77 The aim of this study was to develop a method by which antibiotic resistance in PA can be 78 quantified by calculating a relative resistance index (RRI). This would provide a measure of

79 the total antibiotic resistance of an organism at a given time point, using multiple antibiotic

80 classes (and multiple agents within each of those classes). The RRI would also allow tracking

81 of changes in AS over time. To determine the potential clinical relevance e of the RRI,

82 correlation with important clinical variables in cystic fibrosis patients, including $\mathrm{FEV}_{1} \%$

83 predicted, number of IV (intravenous) antibiotic days and BMI (body mass index) were

84 assessed.

85

86 
Patients attending the Regional Adult Cystic Fibrosis Centre in Northern Ireland (NI) were identified who met the Leeds definition [9] for chronic colonisation with PA between 2010 and 2011. PA was isolated from CF patients' fresh expectorated sputum in a UKAS accredited NHS Clinical Microbiology laboratory, in accordance with laboratory methodologies, as defined in the UK CF Trust guidelines [Laboratory Standards for Processing Microbiological Samples from People with Cystic Fibrosis] [10].Patients concomitantly colonised with Burkholderia cepacia complex were excluded from the analysis. For each patient, the following information was obtained from laboratory data and the Northern Ireland Port CF registry: date of birth, date of first culture of PA, date first defined as chronic PA (Leeds definition), $\mathrm{FEV}_{1} \%$ predicted at annual review 2011, BMI at annual review 2011, total number of IV antibiotic days for 2010 and 2011. Annual review values were used instead of other hospital encounters as these were felt to provide values that were more reflective of the patients' baselines and less likely to be influenced by pulmonary exacerbations. Patients were also classified as to whether they cultured a distinct clonal type commonly isolated in NI, as defined by service genotyping analysis performed by Public Health England, or another unique genotype strain of PA.

104 Patients with CF, especially those from whom organisms are persistently isolated, are seen 105 often at the clinic and sputum is sent to the laboratory at every visit. Antibiotic sensitivities, normally performed on one mucoid and one non-mucoid isolate per calendar month, were estimated using the disc method. For each antibiotic, a report of sensitive (S), intermediate resistance (I) or resistant (R) was issued. During the study period 2010 to 2011, Clinical and

109 Laboratory Standards Institute (CLSI) zone size criteria were applied [11]

110 For each patient, all cultures of PA for which antibiotic sensitivity data was available during 111 the study period $(2010-2011)$ were allocated a score of 0 for susceptible, 0.5 for 
112 intermediate resistance or 1 for resistant for each of the antibiotics listed above. An overall

113 RRI was calculated by adding the means scores for each antibiotic together and dividing by

114 the number of antibiotics (eight in our analysis), giving a minimum RRI of 0 (fully sensitive)

115 and a maximum RRI of 1 (pan-resistant). For the purpose of the study, for each patient we

116 used the mean value of all cultures over the two-year period, to maximise data utilisation and

117 provide a summary measure of resistance over the two-year period.

118 All statistical analyses were performed using SPSS v. 19.0 (IBM Corp., Armonk, NY, USA).

119 Differences between median RRIs between 2010 and 2011 were assessed using Wilcoxon

120 signed rank test. Differences in median RRIs between i) mucoid and non-mucoid PA cultures

121 ii) strain type (LES [Liverpool Epidemic Strain] or unique strain) and iii) gender were

122 assessed using the Mann-Whitney test. Correlation of RRIs with clinical variables (age,

123 duration of chronic PA colonisation, number of IV days 2010-2011, FEV $1 \%$ predicted and

124 BMI at annual review 2011) was assessed using Spearman's Rank correlation. 


\section{RESULTS}

129 Eighty-five patients met the inclusion criteria. As illustrated in Figure 1, the median RRIs

130 were significantly higher for non-mucoid (0.24, IQR $0.10-0.43)$ than mucoid $(0.06$, IQR

$1310.01-0.13)$ PA $(\mathrm{p}<0.001)$. Median RRIs did not differ significantly between 2010 and 2011

132 for non-mucoid $(p=0.14)$ or mucoid PA $(p=0.87)$. Median RRIs were significantly higher

133 for females $(0.26$, IQR $0.13-0.54)$ than males $(0.18$, IQR $0.07-0.37)$ for non-mucoid PA

134 only $(\mathrm{p}=0.03)$. $)$. There was no significant difference between median RRIs in those

135 identified as having the distinct clonal type compared to those with unique PA strains for

136 non-mucoid $(\mathrm{p}=0.14)$ or mucoid PA $(\mathrm{p}=0.55)$. In patients with the aforementioned clonal

137 type, RRIs ranged from 0.00 to 0.75 .

There was a moderate positive correlation of RRIs with age and number of IV days for nonmucoid PA, as detailed in Table 1(A) and (B), whilst in comparison, this was not case for mucoid strains. Figure 2 shows a scatterplot of mean relative resistance indices over time

142 period 2010-2011 against $\mathrm{FEV}_{1} \%$ predicted at annual review 2011. RRI was not significantly

143 correlated with duration of colonisation with chronic PA, typing (clonal vs other strain) or

144 BMI. RRIs for mucoid PA did not correlate significantly with any of the clinical variables 145 examined.

147 Table 1 (C) depicts the correlation of resistance scores for individual antibiotics in non148 mucoid PA against $\mathrm{FEV}_{1} \%$ predicted and number of IV antibiotic days. Resistance to 149 meropenem had the strongest negative correlation with $\mathrm{FEV}_{1} \%$ predicted $(\mathrm{r}=-0.52, \mathrm{p}<$ $150 \quad 0.001)$, followed by ceftazidime $(-0.44, \mathrm{p}<0.001)$. Resistance to ceftazidime had the 151 strongest positive correlation with number of IV antibiotic days $(0.42, \mathrm{p}<0.001)$, followed

152 by meropenem $(0.41,<0.001)$. Although overall scores for mucoid PA did not correlate with 
$153 \mathrm{FEV}_{1} \%$ predicted, significant negative correlation was observed with the score for

154 ceftazidime, meropenem, tazocin and tobramycin in mucoid PA, the strongest and most

155 significant being for tobramycin $(-0.37, \mathrm{p}=0.003)$, followed by meropenem $(-0.31, \mathrm{p}=$ $1560.017)$.

157

158 
161 Chronic infections behave very differently. Although AS is widely used in treating acute

162 infections, there have been few attempts to develop algorithms in CF and elsewhere, which

163 define AS in terms of (i) the totality of antibiotic resistance of an organism at a given time

164 point, over multiple classes/agents within each class or (ii) the change in AS over time

165 reflecting an evolving resistome of PA. But what metrics are optimal for fulfilment of these

166 criteria of such a novel marker/index? In our RRI model, we have proposed the equal

167 weighting of AS across eight antibiotic agents from four antibiotic classes

169 Correlation with clinical parameters were as follows. As expected, RRIs were higher for nonmucoid PA, as strains with mucoid phenotype are protected from the selective pressure imposed by antibiotics. Consequently, it seemed appropriate to analyse correlation with clinical variables separately for mucoid and non-mucoid PA. Our findings show that RRI for PA displays significant correlation with other clinical variables in $\mathrm{CF}$, especially for nonmucoid PA. Total RRIs (i.e. against all antibiotics tested) were positively correlated with number of IV antibiotic days. Figure 2 shows the relationship when RRI is plotted against FEV1 values. It should be noted that from these data, we cannot state that poorer lung

177 function is caused by increasing RRI values and consequently this is not a cause and effect

178 relationship. We are not proposing that RRI is a predictor or cause of lung function, but that

179 the two show a moderate association, most likely due to those with poorer lung function 180 requiring more antibiotic therapy which in turn drives antibiotic resistance, with a resulting

181 increase in RRI values. One surprising finding was the lack of an association between

182 duration of chronic PA colonisation and RRIs, with the number of IV antibiotics days

183 appearing to be a more important contributor to resistance than duration. Median RRIs were 
higher for females than males. This is unsurprising as female patients had more IV antibiotic days, which is likely to have driven antibiotic resistance. Although not recorded in our study, females are also likely to have received more intensive nebulised and oral antibiotic therapy, due to the observation that females tend to experience earlier PA infection and poorer outcomes [12]. RRIs did not differ significantly between patients with the clonal strain compared to those with unique strains, and there was a wide range of RRIs within those with the clonal strain, suggesting considerable heterogeneity in its resistome.

As regards resistance to individual antibiotics, when scores for individual antibiotic resistance are analysed, it appears that meropenem resistance is most strongly associated with a lower $\mathrm{FEV}_{1}$, followed by ceftazidime. The reason for the strong association of meropenem resistance with adverse $\mathrm{FEV}_{1} \%$ predicted is unclear; however it is most likely that this is a reflection of meropenem being reserved for more unwell individuals who may have a lower $\mathrm{FEV}_{1} \%$ predicted, leading to these individuals developing greater meropenem resistance.

199 This relationship requires further understanding and is currently being investigated. As carbapenem resistance is a particularly concerning finding, it may be pertinent to consider weighting meropenem resistance more highly in future RRI models. quality information for targeted questions, and tailored accordingly in terms of its design, depending on what information is required. . Measurement of the gradient of the linear line of best fit, would give an indication of the rate of change in AS ( $\triangle \mathrm{AS})$, which is driven by acquisition of antibiotic resistance and related antibiotic resistance mutation frequency, thus

208 the higher the $\Delta \mathrm{AS}$ value, the faster the development of resistance. Such a marker could be 
extrapolated to potentially estimate future antibiotic resistance development and thus chart certain windows of opportunity, for example, escalation of treatment.

212 Overall, it is probable that individuals with lower FEV1\% predicted, who may be more

213 unwell, require more IV days and therefore develop more resistant forms of PA. However, it

214 is should also be considered that more resistant strains of PA may be predictive of poorer

215 outcomes. Regardless, it appears that greater antibiotic resistance is a marker of lower FEV1

$216 \%$ predicted. In a study of over $4000 \mathrm{CF}$ patients, Ren et al [8] found that mean FEV1

217 decline did not change significantly after Multiple antibiotic-resistant Pseudomonas

218 aeruginosa (MARPA) detection. Consequently they perceived that multi-antibiotic resistance

219 was marker of severe disease, but did not in itself contribute to more rapid decline in FEV1.

220

221 In summary, the advantages of objectively and quantitatively examining AS over time via the

222 RRI marker, within the context of chronic PA colonisation include the ability to (i) succinctly

223 define with a single value how resistant an organism is in its entirety over several classes of

224 antibiotics, at a given time point. Such information would be useful to clinicians in helping

225 to determine suitable antibiotic management strategies, e.g antibiotic cycling, (ii) monitor the

226 rate at which antibiotic resistance is developing, (iii) provide evidence relating to the

227 introduction/elimination of particular strains of PA, as seen with markedly different RRI

228 scores appearing/disappearing, with implications for infection control/antibiotic management,

229 A rapidly changing RRI could also indicate the need to re-type the PA to assess if a new

230 strain has been introduced. This could provide guidance as to when typing is warranted, as

231 most centres do not regularly type PA due to financial restraints, and (iv) estimate windows

232 of opportunity where antibiotic resistance is important e.g. deteriorating lung health and

233 antibiotic allergy. 
235 This work represents an advance in biomedical science because to date, no such cumulative approach has been described. This novel Relative Resistance Index (RRI) marker may be a useful tool in forming a cumulative determination of antimicrobial susceptibility over several antibiotics through time, with useful benefits in quantifying antimicrobial resistance to clinicians and other healthcare professionals.

What is known about this subject:

- Antimicrobial resistance has now emerged as a major threat to public health at a national and international level

- Routine clinical microbiology laboratories test antimicrobial resistance individually for each antibiotic

- There is no laboratory biomarker or algorithm that attempts to link cumulative antimicrobial susceptibility for several antibiotics simultaneously into a single holistic marker, for ease of interpretation amongst clinical staff

What this paper adds:

- The proposed Relative Resistance Index (RRI) is a means of combining single antimicrobial susceptibilities into a single index, that is easily interpreted by clinical staff

- RRI provides a flexible and quantitative measure of resistance which allows statistical correlation of resistance with other clinical variables. In the patients in our study, RRI showed a moderate association with lung function and number of IV antibiotic days.

- Within cystic fibrosis (CF), where antimicrobial resistance has a major influence on patient morbidity and mortality, the RRI could be extrapolated to potentially estimate future antibiotic resistance development and thus chart certain clinical windows of opportunity, for example, escalation of treatment. 
1. Fodor AA, KlemER, Gilpin DF et al. The adult cystic fibrosis airway microbiota is stable over time and infection type, and highly resilient to antibiotic treatment of exacerbations. PloS one 2012; 7:, e45001.

2. Doring G. Conway SP, Heijerman HG. et al. Antibiotic therapy against Pseudomonas aeruginosa in cystic fibrosis: a European consensus. Eur. Respir. J 2000; 16: 749767.

3. Høiby N. Recent advances in the treatment of Pseudomonas aeruginosa infections in cystic fibrosis. BMC Medicine 2011; 9: 32.

4. Pritt B, O’Brien L, Winn W. Mucoid Pseudomonas in cystic fibrosis. Am. J. Clin Path. 2007; 128: 32-34.

5. Kadry AA, Serry FM, El-Ganiny AM, El-Baz AM. Integron occurrence is linked to reduced biocide susceptibility in multidrug resistant_Pseudomonas_aeruginosa. Br J Biomed Sci. 2017;;74):78-84..

6. Champion AC, Houston NK, Bradbury RS, Reid DW. Preliminary feasibility and modelling of a liquid matrix Dictyostelium discoideum virulence assay for_Pseudomonas_aeruginosa. Br J Biomed Sci. 2016;73:51-55.

7. Falagas ME, Koletsi PK and Bliziotis IA. The diversity of definitions of multidrugresistant (MDR) and pandrug-resistant (PDR) Acinetobacter baumannii and Pseudomonas aeruginosa. J. Med. Microbiol 2006; 55: 1619-1629.

8. Ren CL, Konstan MW, Yegin A. et al. W. Multiple antibiotic-resistant Pseudomonas aeruginosa and lung function decline in patients with cystic fibrosis. J. Cystic Fibrosis 2012; 11: 293-299.

9. Proesmans M, Balinska-Miskiewicz W, Dupont L. et al. Evaluating the "Leeds criteria" for Pseudomonas aeruginosa infection in a cystic fibrosis centre. Eur. Respir. J 2006; 27: 937-943.

10. Anon. Laboratory Standards for Processing Microbiological Samples from People with Cystic Fibrosis. https://www.cysticfibrosis.org.uk/the-work-we-do/clinicalcare/consensus-documents [Last accessed 25 May 2017]

11. Anon. Clinical and Laboratory Standards Institute. 2005. Performance standards for antimicrobial susceptibility testing; 15th informational supplement. CLSI/NCCLS M100-S15. Clinical and Laboratory Standards Institute, USA.

12. Demko CA, Byard PJ. and Davis PB. Gender differences in cystic fibrosis: Pseudomonas aeruginosa infection. J. Clin Epidemiol 1995; 48: 1041-1049. 
320 Figure 1. Box plot of mean relative resistance indices for non-mucoid compared with mucoid

321 Pseudomonas aeruginosa for cultures taken over 2010-2011.



322

323

324

325

326 
327 Figure 2: Scatterplot of mean relative resistance indices over time period 2010-2011 328 against $\mathrm{FEV}_{1} \%$ predicted at annual review 2011.

329

330

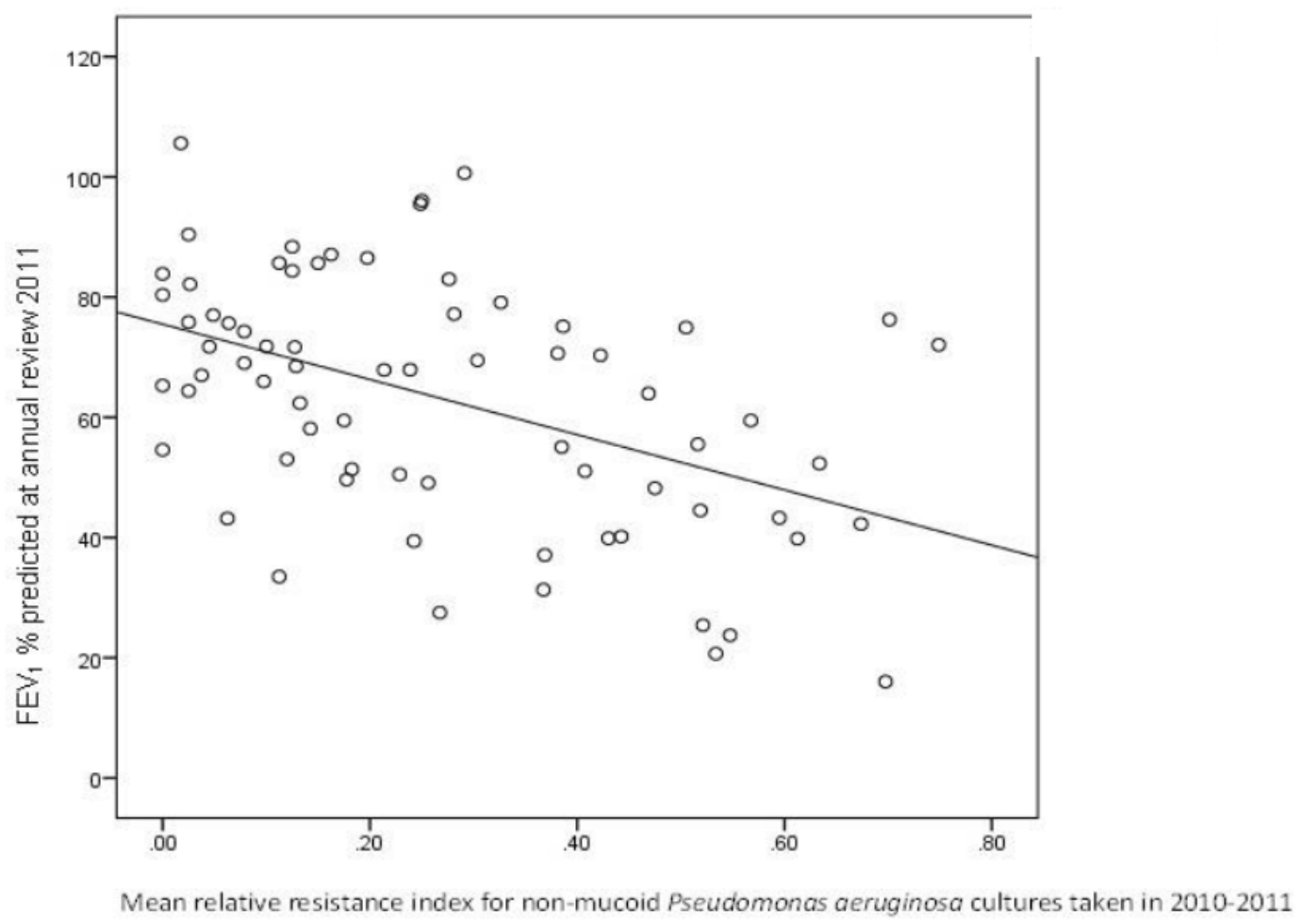


Table 1.

A. Correlation between relative resistance indices (mean over 2010 and 2011) and clinical variables for non-mucoid and mucoid Pseudomonas aeruginosa. For non-mucoid PA, relative resistance index correlates positively with age and number of intravenous antibiotic days, and negatively with $\mathrm{FEV}_{1} \%$ predicted.

Spearman's Rank Correlation - Correlation Coefficient (Sig. 2 tailed)

Non-mucoid PA

RRI 2010-2011
Mucoid PA

RRI $2010-2011$

\begin{tabular}{lll}
\hline Age & & \\
Duration of Chronic PA & $0.24(0.04)^{*}$ & $0.036(0.77)$ \\
colonisation & $0.06(0.62)$ & $0.090(0.47)$ \\
Number of IV antibiotic days & $0.41(<0.001)^{\star *}$ & $0.122(0.33)$ \\
$2010-2011$ & $-0.014(0.91)$ \\
FEV1 \% predicted 2011 & $-0.44(<0.001)^{\star *}$ & $-0.081(0.53)$ \\
(annual review) & $-0.09(0.46)$ &
\end{tabular}

B. Multiple linear regression model for predicting mean relative resistance index for non-mucoid Pseudomonas aeruginosa in 2011.

\begin{tabular}{llll} 
& $\begin{array}{l}\text { Unstandardized } \\
\text { co-efficient (B) }\end{array}$ & $\begin{array}{l}\mathrm{t} \text { test statistic for } \\
\text { co-efficient }\end{array}$ & Significance \\
\hline Gender & 0.960 & 1.850 & 0.072 \\
Age & 0.063 & 1.823 & 0.076 \\
Typing (clonal strain or other) & -0.694 & -1.304 & 0.200 \\
Duration of chronic PA colonisation & 0.000 & 0.687 & 0.496 \\
Total IV days 2010-2011 & 0.018 & 2.393 & $0.021^{*}$ \\
\hline
\end{tabular}

\section{Correlation between resistance scores for individual antibiotics and FEV $1 \%$ predicted 2011 and total intravenous antibiotic days 2010-2011.}

Spearmans Rank Correlation - Correlation Coefficient (Sig. 2 tailed)

FEV1 \% predicted 2011 Total IV antibiotic days $2010-2011$

\begin{tabular}{llll}
\hline & Aztreonam & $-0.27(0.02)^{\star}$ & $0.35(0.001)^{\star *}$ \\
& Ceftazidime & $-0.44(<0.001)^{\star *}$ & $0.428(<0.001)^{\star *}$ \\
& Ciprofloxacin & $-0.13(0.298)$ & $0.14(0.21)$ \\
Resistance score for & Colistin & $-0.16(0.196)$ & $0.09(0.41)$ \\
individual antibiotics & Meropenem & $-0.52(<0.001)^{\star *}$ & $0.41(<0.001)^{\star *}$ \\
Non-mucoid PA 2010-2011 & Tazocin & $-0.367(0.001)^{\star *}$ & $0.41(<0.001)^{\star *}$ \\
& Temicillin & $-0.20(0.10)$ & $0.29(0.011)^{*}$ \\
& Tobramycin & $-0.42(<0.001)^{\star *}$ & $0.258(0.03)^{\star}$ \\
\hline
\end{tabular}

